\title{
Weak lensing mass reconstruction using wavelets
}

\author{
J.-L. Starck, S. Pires, and A. Réfrégier
}

\author{
DAPNIA/SEDI-SAP, Service d'Astrophysique, CEA-Saclay, 91191 Gif-sur-Yvette Cedex, France \\ e-mail: jstarck@cea.fr
}

Received 5 March 2005 / Accepted 18 January 2006

\begin{abstract}
This paper presents a new method for the reconstruction of weak lensing mass maps. It uses the multiscale entropy concept, which is based on wavelets, and the False Discovery Rate (FDR) which allows us to derive robust detection levels in wavelet space. We show that this new restoration approach outperforms several standard techniques currently used for weak shear mass reconstruction. This method can also be used to separate E and B modes in the shear field, and thus test for the presence of residual systematic effects. We concentrate on large blind cosmic shear surveys, and illustrate our results using simulated shear maps derived from $N$-Body $\Lambda C D M$ simulations (Vale \& White 2003) with added noise corresponding to both ground-based and space-based observations.
\end{abstract}

Key words. cosmology: dark matter - methods: data analysis - gravitational lensing

\section{Introduction}

Weak Gravitational Lensing provides a unique method to map directly the distribution of dark matter in the universe (Bartelmann \& Schneider 1999; Mellier 1999; Van Waerbeke et al. 2001; Mellier 2002; Refregier 2003). This method is based on the weak distortions that lensing induces in the images of background galaxies as light travels through intervening structures. This method is now widely used to map the mass of clusters and superclusters of galaxies and to measure the statistics of the cosmic shear field on large scales.

Ongoing efforts are made to improve the detection of cosmic shear on existing telescopes and future instruments dedicated to survey cosmic shear are planned. Several methods are used to derive the lensing shear from the shapes of background galaxies. But the shear measurements obtained are always noisy, and when it is converted into a map of the projected mass $\kappa$, the result is dominated by the noise.

Several methods have been devised to reconstruct the projected mass distribution from the observed shear field. The first non-parametric mass reconstruction was proposed by Kaiser \& Squires (1993) and further improved by Bartelmann (1995); Kaiser (1995); Schneider \& Seitz (1995); Squires \& Kaiser (1996). These methods are based on linear inversion methods based on smoothing with a fixed kernel. Non-linear reconstruction methods were proposed using a maximum likelihood approach (Squires \& Kaiser 1996; Bartelmann et al. 1996; Seitz et al. 1998) or using the maximum entropy method (Bridle et al. 1998; Marshall et al. 2002).

In this paper, we describe a method for weak lensing mass reconstruction based on a wavelet decomposition. We use an iterative filtering method with a multiscale entropy regularisation to filter the noise. We discuss how this decomposition and regularisation functional is particularly well adapted to this problem. In the process, we identify significant wavelet coefficients using the False Discovery Rate method (Miller et al. 2001; Hopkins et al. 2002) and show how this is superior to the standard $n \sigma$ thresholding. The FDR method adapts its threshold to the features of the data. We concentrate on large blind cosmic shear surveys and use the ray-tracing simulations of Vale \& White (2003) to test our results. We compare the performance of our method to Gaussian and Wiener filtering for the reconstruction of the mass field in these simulations. We consider conditions similar to both ground-based and space-based cosmic shear surveys. We also discuss how our method differs from other methods based on the maximum entropy prior.

In Sect. 2, we present the weak shear mass reconstruction problem. The earlier methods which have been proposed to reconstruct the mass map are described in Sect. 3. Section 4 presents the Multiscale Entropy method and explains why it is a good alternative to standard methods. We also propose a modification of the Multiscale Entropy, we use the False Discovery Rate (FDR) method for detecting the significant wavelet coefficients. A set of experiments designed to test our method are described Sect. 5. Our conclusions are summarized in Sect. 6.

\section{Weak lensing mass reconstruction}

\subsection{Weak lensing}

In weak lensing surveys, the shear $\gamma_{i}(\theta)$ with $i=1,2$ is derived from the shapes of galaxies at positions $\theta$ in the image. The shear field $\gamma_{i}(\theta)$ can be written in terms of the lensing potential $\psi(\theta)$ as (see e.g. Bartelmann \& Schneider 1999)

$$
\begin{aligned}
& \gamma_{1}=\frac{1}{2}\left(\partial_{1}^{2}-\partial_{2}^{2}\right) \psi \\
& \gamma_{2}=\partial_{1} \partial_{2} \psi,
\end{aligned}
$$

where the partial derivatives $\partial_{i}$ are with respect to $\theta_{i}$. The convergence $\kappa(\theta)$ can also be expressed in terms of the lensing potential as

$\kappa=\frac{1}{2}\left(\partial_{1}^{2}+\partial_{2}^{2}\right) \psi$ 


\begin{tabular}{llllllllll}
\hline \hline 0.0 & 0.1 & 0.2 & 0.3 & 0. & 0.0 & 0.1 & 0.2 & 0.3 & 0.
\end{tabular}
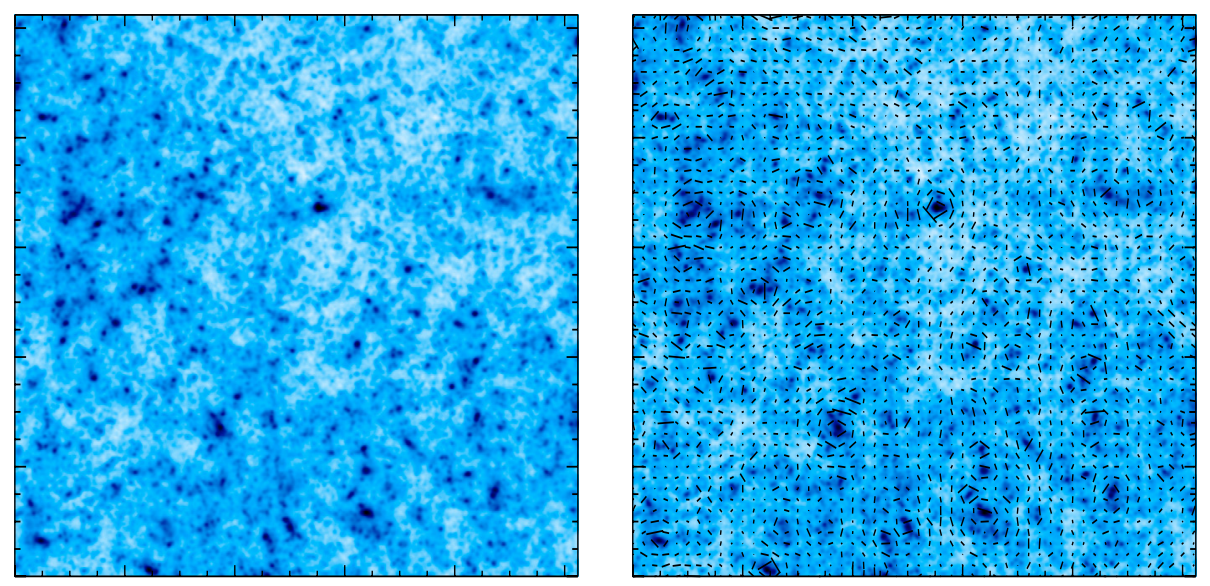

Fig. 1. Left: simulated convergence map from (Vale \& White 2003) for a $\Lambda$ CDM model. The region shown is $2 \times 2$ square degree. Right: shear map superimposed on the convergence map, and right shear map. The size and direction of each line gives the amplitude and position angle of the shear at this location on the sky.

and is related to the surface density $\Sigma(\theta)$ projected along the line of sight by

$\kappa(\theta)=\frac{\Sigma(\theta)}{\Sigma_{\text {crit }}}$

where the critical surface density is given by

$\Sigma_{\text {crit }}=\frac{c^{2}}{4 \pi G} \frac{D_{s}}{D_{l} D_{l s}}$

and $G$ is Newton's constant, $c$ is the speed of light and $D_{s}, D_{l}$ and $D_{l s}$ are the angular-diameter distances between the observer and the galaxies, the observer and the lens, and the lens and the galaxies. In practice, the galaxies are not at a fixed redshift, and the expression for $\kappa$ is an average of the redshift of the galaxies (see e.g. Bartelmann 1995). The lensing effect is said to be weak or strong if $\kappa \ll 1$ or $\kappa \gtrsim 1$, respectively.

The left panel of Fig. 1 shows a simulated convergence map derived from ray-tracing through $\mathrm{N}$-body cosmological simulations performed by Vale \& White (2003). The cosmological model is taken to be a concordance $\Lambda \mathrm{CDM}$ model with parameters $\Omega_{M}=0.3, \Omega_{\Lambda}=0.7, h=0.7$ and $\sigma_{8}=0.8$. The simulation contains $512^{3}$ particles with a box size of $300 h^{-1} \mathrm{Mpc}$. The resulting convergence map covers $2 \times 2$ degrees with $1024 \times$ 1024 pixels and assume a galaxy redshift of 1 . The overdensities correspond to the haloes of groups and clusters of galaxies. The rms value of $\kappa$ binned in 0.12 arcmin pixels is $\sigma_{\kappa}=0.023$. The typical values of $\kappa$ are thus of the order of a few percent, apart from the core of massive halos (see Fig. 1). The weak lensing condition therefore holds in most regions of the sky and will be assumed throughout this paper.

\subsection{Mass inversion}

The weak lensing mass inversion problem consists of reconstructing the projected (normalized) mass distribution $\kappa(\theta)$ from the measured shear field $\gamma_{i}(\theta)$ by inverting Eqs. (1) and (2). (Magnification information can also be used to improve the reconstruction (see Bridle et al. 1998), but is typically more noisy than the shear measurements and has not been considered in this paper). For this purpose, we take the Fourier transform of these equations and obtain

$\hat{\gamma}_{i}=\hat{P}_{i} \hat{\kappa}, \quad i=1,2$

where the hat symbol denotes Fourier transforms and we have defined $k^{2} \equiv k_{1}^{2}+k_{2}^{2}$ and

$\hat{P}_{1}(\boldsymbol{k})=\frac{k_{1}^{2}-k_{2}^{2}}{k^{2}}$
$\hat{P}_{2}(\boldsymbol{k})=\frac{2 k_{1} k_{2}}{k^{2}}$,

with $\hat{P}_{1}\left(k_{1}, k_{2}\right) \equiv 0$ when $k_{1}^{2}=k_{2}^{2}$, and $\hat{P}_{2}\left(k_{1}, k_{2}\right) \equiv 0$ when $k_{1}=0$ or $k_{2}=0$.

The shear map $\gamma_{i}$ can be calculated from the convergence map $\kappa$ using these expressions. The right panel of Fig. 1, shows the shear field associated with the simulated convergence field. As is customary, the direction and size of the line segment represent the orientation and amplitude of the shear. The rms shear in the 0.12 amin pixels of the resulting map is $\sigma_{\gamma}=\left\langle\gamma_{1}^{2}+\gamma_{2}^{2}\right\rangle^{\frac{1}{2}} \simeq 0.023$.

Note that to recover $\kappa$ from $\gamma_{1}$ (resp. $\gamma_{2}$ ), there is a degeneracy when $k_{1}^{2}=k_{2}^{2}$ (resp. when $k_{1}=0$ or $\left.k_{2}=0\right)$. To recover $\kappa$ from both $\gamma_{1}$ and $\gamma_{2}$, there is a degeneracy only when $k_{1}=k_{2}=0$. Therefore, the mean value of $\kappa$ cannot be recovered from the shear maps. This is a special instance of the well known mass-sheet degeneracy in the weak lensing reconstruction if only shear information is available (see e.g. Bartelmann 1995, for a discussion).

In practice, the observed shear $\gamma_{i}$ is obtained by averaging over a finite number of galaxies and is therefore noisy. The relations between the observed data $\gamma_{1 b}, \gamma_{2 b}$ binned in pixels of area $A$ and the true mass map $\kappa$ are given by:

$\gamma_{i b}=P_{i} * \kappa+N_{i}$

where $N_{1}$ and $N_{2}$ are noise contributions with zero mean and standard deviation $\sigma_{n} \simeq \sigma_{\epsilon} / \sqrt{N_{\mathrm{g}}}$, where $N_{\mathrm{g}}=n_{\mathrm{g}} A$ is the average number of galaxies in a pixel and $n_{\mathrm{g}}$ is the average number of galaxies per $\operatorname{arcmin}^{2}$. The rms shear dispersion per 
galaxy $\sigma_{\epsilon}$ arises both from measurement errors and the intrinsic shape dispersion of galaxies. In this analysis, we will assume $\sigma_{\epsilon} \simeq 0.3$ as is approximately found for ground-based and space-based weak lensing surveys. Typical values for the surface density of usable galaxies for weak lensing are

- $n_{\mathrm{g}}=20 \mathrm{gal} / \mathrm{arcmin}^{2}$ for ground-based surveys.

- $n_{\mathrm{g}}=100 \mathrm{gal} / \mathrm{arcmin}^{2}$ for space-based surveys.

From the central limit theorem, this means that for pixels with $A \gtrsim 1 \mathrm{amin}^{2}$, the noise $N_{i}$ is, to a good approximation, Gaussian in both cases and is uncorrelated (see Marshall et al. 2002, for a direct treatment of individual galaxy shears using the MEM method).

\subsection{The inverse filter: $E$ and $B$ mode}

We can easily derive an estimation of the mass map by inverse filtering by noticing that

$\hat{P}_{1}^{2}+\hat{P}_{2}^{2}=1$.

The least square estimator $\hat{\tilde{\kappa}}_{l}^{(E)}$ of the convergence $\hat{\kappa}$ in the Fourier domain is:

$\hat{\tilde{\kappa}}_{l}^{(E)}=\hat{P}_{1} \hat{\gamma}_{1 b}+\hat{P}_{2} \hat{\gamma}_{2 b}$.

The relation between this estimator and the true mass map is $\hat{\tilde{\kappa}}_{l}^{(E)}=\hat{\kappa}+\hat{N}$, where $\hat{N}=\hat{P}_{1} \hat{N}_{1}+\hat{P}_{2} \hat{N}_{2}$.

Just as any vector field, the shear field $\gamma_{i}(\theta)$ can be decomposed into a gradient, or electric $(E)$, component, and a curl, or magnetic $(B)$, component. Because the weak lensing arises from a scalar potential (the Newtonian potential), it can be shown that weak lensing only produces $E$-modes. On the other hand, residual systematics arising from imperfect correction of the instrumental PSF or telescope aberrations, generally generates both $E$ and $B$ modes. The presence of $B$-modes is thus used to test for the presence of residual systematic effects in current weak lensing surveys.

The decomposition of the shear field into each of these components can be easily performed by noticing that a pure $E$-mode can be transformed into a pure $B$ mode by a rotation of the shear by $45^{\circ}: \gamma_{1} \rightarrow-\gamma_{2}, \gamma_{2} \rightarrow \gamma_{1}$. As a result, we can form the following estimator for the $B$-mode "convergence" field

$\hat{\tilde{\kappa}}_{l}^{(B)}=\hat{P}_{2} \hat{\gamma}_{1 b}-\hat{P}_{1} * \hat{\gamma_{2} b}$,

and check that it is consistent with zero in the absence of systematics.

As follows from Eq. (8), the noise $N^{(E)}$ and $N^{(B)}$ in $\tilde{\kappa}_{l}^{(E)}$ and $\tilde{\kappa}_{l}^{(B)}$ is still Gaussian and uncorrelated. The inverse filtering does not amplify the noise, but $\tilde{\kappa}_{l}^{(E)}$ and $\tilde{\kappa}_{l}^{(B)}$ may be dominated by the noise if $N^{(E)}$ and $N^{(B)}$ are large, which is the case in practice. Figure 2 shows the reconstructed mass map using Eq. (9) when a realistic Gaussian noise has been added to the shear maps plotted in Fig. 1 right. As expected, it is dominated by noise. This has motivated the development of different methods in the past which we describe below.

\section{Earlier mass inversion methods}

\subsection{Linear filtering}

The standard method (Kaiser \& Squires 1993) consists in convolving the noisy mass map $\widetilde{\kappa}_{l}^{(E)}$ with a Gaussian window $G$ with standard deviation $\sigma_{\mathrm{G}}$ :

$\tilde{\kappa}_{\mathrm{G}}^{(E)}=G * \tilde{\kappa}_{l}^{(E)}=G * P_{1} * \gamma_{1 b}+G * P_{2} * \gamma_{2 b}$.

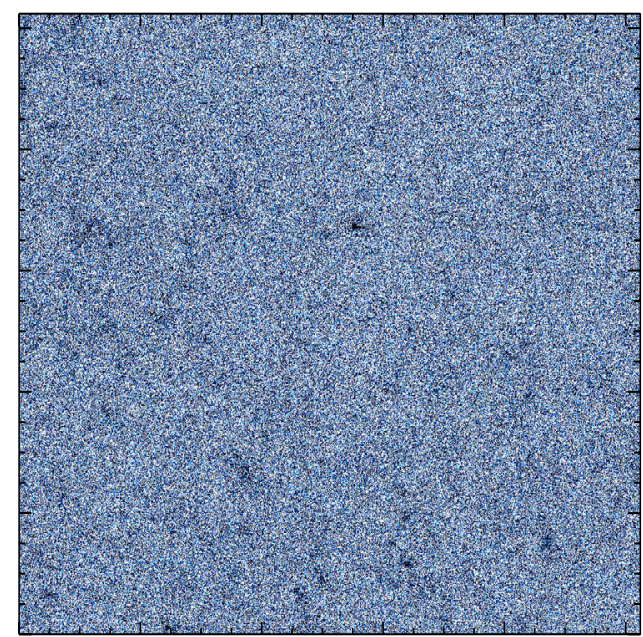

Fig. 2. In the previous region of $2 \times 2$ square degrees, noisy mass map $\kappa_{l}^{(E)}$ for the same simulation with $n_{\mathrm{g}}=100 \mathrm{gal} / \mathrm{arcmin}^{2}$, corresponding to space-based observations. Even in this case, the unfiltered mass map is dominated by noise.

The quality of the resulting estimation depends strongly on the value of $\sigma_{\mathrm{G}}$. Figure 3 shows the variation of the error between the original mass map $\kappa$ shown in Fig. 1 and the filtered mass map $\tilde{\kappa}_{\mathrm{G}}^{(E)}$. For this simulation, the optimal value of $\sigma_{\mathrm{G}}$ lies between 5 and 10 pixels ( 1 pixel $=0.12$ arcmin) for space observations (i.e. $\left.n_{\mathrm{g}}=100 \mathrm{gal} / \mathrm{amin}^{2}\right)$ and lies between 20 and 25 pixels for ground observations (i.e. $n_{\mathrm{g}}=20 \mathrm{gal} / \mathrm{amin}^{2}$ ).

An alternative to Gaussian filtering is the Wiener filtering, obtained by assigning the following weight to each $k$-mode

$w(k)=\frac{|\hat{\kappa}(k)|^{2}}{|\hat{\kappa}(k)|^{2}+|\hat{N}(k)|^{2}}$.

Where $|\hat{\kappa}(k)|^{2}$ is a model of the true convergence power spectrum and is in practice derived from the data. Wiener filtering is known to be optimal when both the signal and the noise are a realization of a Gaussian Random Field. As can be seen from Fig. 1, this assumption is not valid for weak lensing mass maps which display non-Gaussian features such as galaxy clusters, groups and filaments. Even in this case, Wiener Filtering nevertheless leads to reasonable results, generally better than the simple Gaussian filtering.

\subsection{Maximum entropy method}

The Maximum Entropy Method (MEM) is well-known and widely used in image analysis in astronomy (see Bridle et al. 1998; Starck et al. 2001; Marshall et al. 2002; Starck \& Murtagh 2002 , for a full description). It considers both the data and the solution as probability density functions and finds the solution using a Bayesian approach and adding a prior (the entropy) on the solution. Several definitions of entropy exist. The most common is the definition proposed in Gull \& Skilling (1991):

$H_{\mathrm{g}}(\kappa)=\sum_{x} \sum_{y} \kappa(x, y)-m(x, y)-\kappa(x, y) \ln \left(\frac{\kappa(x, y)}{m(x, y)}\right)$

where $m$ is a model, chosen typically to be a sky background. $H_{\mathrm{g}}$ has a global maximum at $\kappa=m$. MEM does not allow negative values in the solution, which is unnatural for wide field 

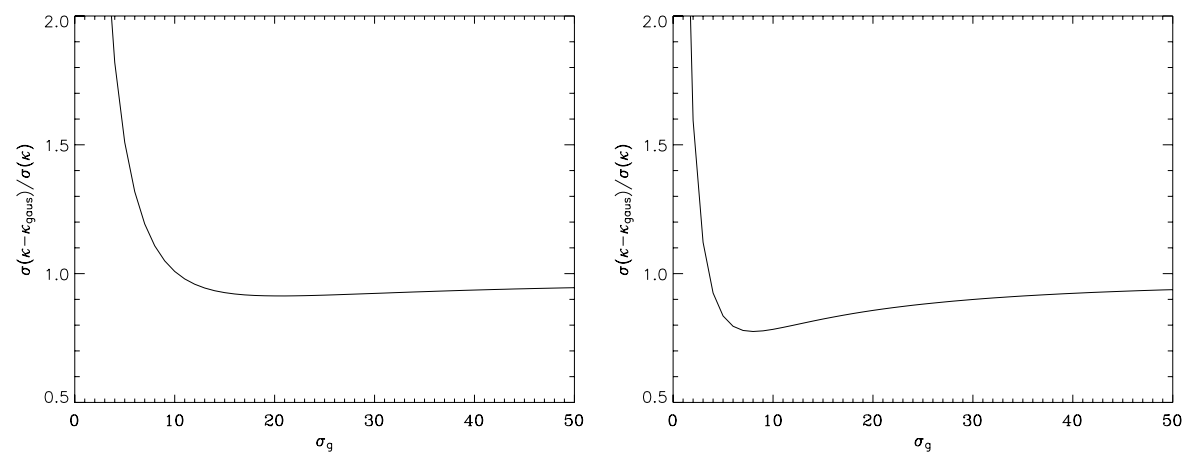

Fig. 3. Reconstruction error as a function of the kernel size $\sigma_{\mathrm{G}}$ (in 0.12 amin pixels) for the Gaussian smoothing method, with $n_{\mathrm{g}}=$ $20 \mathrm{gal} / \mathrm{amin}^{2}(l e f t)$ and $n_{\mathrm{g}}=100 \mathrm{gal} / \mathrm{amin}^{2}$ (right). weak shear data or the CMB data, where we measure fluctuations around zero. To overcome this, it has been proposed to replace $H_{\mathrm{g}}$ (Maisinger et al. 2004) by:

$$
\begin{aligned}
H_{+/-}(\kappa)= & \sum_{x} \sum_{y} \psi(x, y)-2 m \\
& -\kappa(x, y) \ln \left(\frac{\psi(x, y)+\kappa(x, y)}{2 m}\right)
\end{aligned}
$$

where $\psi(x, y)=\sqrt{\kappa^{2}(x, y)+4 m^{2}}$. Here $m$ does not play the same role. It is a constant fixed to the expected signal rms.

More generally MEM method presents many drawbacks (Narayan \& Nityananda 1986; Starck et al. 2001) and various refinements of MEM have been proposed over the years (Weir 1992; Bontekoe et al. 1994; Pantin \& Starck 1996; Starck et al. 2001). The last developments have led to the so called Multiscale Entropy (Pantin \& Starck 1996; Starck et al. 2001; Maisinger et al. 2004) which is based on an undecimated isotropic wavelet transform (à trous algorithm) (Starck et al. 1998). It has been shown that the main MEM drawbacks (model dependent solution, oversmoothing of compact objects, ...) disappear in the wavelet framework. A full discussion and comparison between different restoration methods can be found in Starck et al. (2002).

\section{Multiscale entropy restoration}

\subsection{The multiscale entropy}

The Undecimated Isotropic Wavelet Transform (UIWT) decomposes an $n \times n$ image $I$ as a superposition of the form

$I(k, l)=c_{J k, l}+\sum_{j=1}^{J} w_{j, k . l}$,

where $c_{J}$ is a coarse or smooth version of the original image $I$ and $w_{j}$ represents the details of $I$ at scale $2^{-j}$ (see Starck et al. (Starck et al. 1998; Starck \& Murtagh 2002) for details). Thus, the algorithm outputs $J+1$ sub-band arrays of size $n \times n$. We will use an indexing convention such that $j=1$ corresponds to the finest scale (high frequencies). The Multiscale Entropy concept (Pantin \& Starck 1996) consists in replacing the standard MEM prior (i.e. the Gull and Skilling entropy) by a wavelet based prior. The entropy is now defined as

$H(I)=\sum_{j=1}^{J-1} \sum_{k, l} h\left(w_{j, k . l}\right)$

In this approach, the information content of an image is viewed as sum of information at different scales. The function $h$ defines the amount of information relative to a given wavelet coefficient. Several functions have been proposed for $h$ :

- LOG-MSE: The Multiscale Entropy function used in (Pantin \& Starck 1996) (we call it LOG-MSE in the following) is defined by:

$h\left(w_{j, k, l}\right)=\frac{\sigma_{j}}{\sigma_{X}^{2}}\left[w_{j, k, l}-m_{j}-\left|w_{j, k, l}\right| \log \left(\frac{\left|w_{j, k, l}\right|}{K_{m} \sigma_{j}}\right)\right]$

where $\sigma_{X}$ is the total noise standard deviation of the data and $\sigma_{j}$ is the noise standard deviation at scale $j . K_{m}$ is a user-supplied parameter.

- ENERGY-MSE: The entropy can be defined as the function of the square of the wavelet coefficients (Starck et al. 2001):

$h\left(w_{j, k, l}\right)=\frac{w_{j, k, l}^{2}}{\sigma_{j}^{2}}$.

The same multiscale entropy function was also derived in Maisinger et al. (2004).

- NOISE-MSE: In Starck et al. (2001), the entropy is derived using a modeling of the noise contained in the data:

$h\left(w_{j, k, l}\right)=\int_{0}^{\left|w_{j, k, l}\right|} P_{n}\left(\left|w_{j, k, l}\right|-u\right)\left(\frac{\partial h(x)}{\partial x}\right)_{x=u} \mathrm{~d} u$

where $P_{n}\left(w_{j, k, l}\right)$ is the probability that the coefficient $w_{j, k, l}$ can be due to the noise: $P_{n}\left(w_{j, k, l}\right)=\operatorname{Prob}\left(W>\left|w_{j, k, l}\right|\right)$. For Gaussian noise, we have:

$$
\begin{aligned}
P_{n}\left(w_{j, k, l}\right) & =\frac{2}{\sqrt{2 \pi} \sigma_{j}} \int_{\left|w_{j, k, l}\right|}^{+\infty} \exp \left(-W^{2} / 2 \sigma_{j}^{2}\right) \mathrm{d} W \\
& =\operatorname{erfc}\left(\frac{\left|w_{j, k, l}\right|}{\sqrt{2} \sigma_{j}}\right)
\end{aligned}
$$

and

$$
h\left(w_{j, k, l}\right)=\frac{1}{\sigma_{j}^{2}} \int_{0}^{\left|w_{j, k, l}\right|} u \operatorname{erfc}\left(\frac{\left|w_{j, k, l}\right|-u}{\sqrt{2} \sigma_{j}}\right) \mathrm{d} u
$$

LOG-MSE presents an indeterminacy when the wavelet coefficient is equal or close to 0 and the model used in Eq. (15) is somewhat ad hoc. This point was raised in Maisinger et al. (2004). A better choice for the LOG-MSE would be the Herbert and Leaby function (Hebert \& Leahy 1989) (see also the discussion in Sect. 4.4):

$$
h\left(w_{j, k, l}\right) \propto \log \left(1+\frac{\left|w_{j, k, l}\right|}{\sigma_{j}}\right) .
$$


The ENERGY-MSE is quadratic and leads to a strong penalization even for wavelet coefficients with high signal-to-noise ratio. Such penalization terms are known to oversmooth the strongest peaks and should not be used for the weak lensing mass reconstruction. The NOISE-MSE is very close to the $l_{1}$ norm (i.e. absolute value of the wavelet coefficient) when the coefficient value is large, which is known to produce good results for the analysis of piecewise smooth images (Donoho \& Elad 2003). We therefore choose the NOISE-MSE entropy as the most appropriate for the weak lensing reconstruction problem. Figure 5 shows the $l_{1}$ norm penalization function, the ENERGY-MSE and NOISE-MSE. The NOISE-MSE penalization presents a quadratic behavior for small coefficients and a linear one for larger coefficients. More details are given in Sect. 4.4.

\subsection{Significant wavelet coefficients using the FDR}

In Pantin \& Starck (1996), it has been suggested to not apply the regularization on wavelet coefficients which are clearly detected (i.e. significant wavelet coefficients). The new Multiscale Entropy is:

$h_{n}\left(w_{j, k, l}\right)=\bar{M}(j, k, l) h\left(w_{j, k, l}\right)$

where $\bar{M}(j, k, l)=1-M(j, k, l)$, and $M$ is the multiresolution support (Murtagh et al. 1995):

$M(j, k, l)= \begin{cases}1 & \text { if } w_{j, k, l} \text { is significant } \\ 0 & \text { if } w_{j, k, l} \text { is not significant. }\end{cases}$

This describes, in a Boolean way, whether the data contains information at a given scale $j$ and at a given position $(k, l)$. Commonly, $w_{j, k, l}$ is said to be significant if the probability that the wavelet coefficient is due to noise is small, i.e. if $P(\mid W>$ $\left.w_{j, k, l} \mid\right)<\epsilon$, where $P$ is a given noise distribution function. In the case of Gaussian noise, this amount to state that $w_{j, k, l}$ is significant if $\left|w_{j, k, l}\right|>k \sigma_{j}$, where $\sigma_{j}$ is the noise standard deviation at scale $j$, and $k$ is a constant, generally taken between 3 and 5 (Murtagh et al. 1995). With this definition, the number of false detections depends on both the $\epsilon$ value and the image size.

An alternative approach to this detection strategy is the False Discovery Rate method (FDR) (Benjamini \& Hochberg 1995). This technique has recently be introduced for astronomical data analysis (Miller et al. 2001; Hopkins et al. 2002). It allows us to control the average fraction of false detections made over the total number of detections. It also offers an effective way to select an adaptive threshold. The FDR is given by the ratio:

$\mathrm{FDR}=\frac{V_{\mathrm{ia}}}{D_{\mathrm{a}}}$

where $V_{\mathrm{ia}}$ are the number of pixels truly inactive declared active and $D_{\mathrm{a}}$ are the number of pixels declared active.

This procedure controlling the FDR specifies a rate $\alpha$ between 0 and 1 and ensures that, on average, the FDR is no bigger than $\alpha$ :

$E(\mathrm{FDR}) \leq \frac{T_{i}}{V} \cdot \alpha \leq \alpha$.

The unknown factor $\frac{T_{i}}{V}$ is the proportion of truly inactive pixels. A complete description of the FDR method can be found in Miller et al. (2001). In Hopkins et al. (2002), it has been shown that the FDR outperforms standard methods for sources detection.
Here, we use the FDR method; at each resolution level $j$ of the decomposition. We derive a detection threshold $T_{j}$ (from a $\alpha_{j}$ value). We have chosen to take a different $\alpha$ value per scale. To fix a $\alpha$ value per scale, we used The Receiver Operating Characteristic (ROC) (Genovese \& Eddy 1997) curves in order to quantify the quality of the detection at a given scale for different $\alpha$ values. We found that the $\alpha_{j}$ value must increase with scale following the relation: $\alpha_{j}=\alpha_{0} * 2^{j}$ for the spatial observations and $\alpha_{j}=\alpha_{0} *(1.7)^{j}$ for the ground observations where $\alpha_{0}=0.0125$. We then consider a wavelet coefficient $w_{j, k, l}$ as significant if its absolute value is larger than $T_{j}$.

\subsection{Multiscale entropy restoration}

Assuming Gaussian noise, the Multiscale Entropy restoration method lead to the minimization of the functional,

$J(\tilde{\kappa})=\frac{\left\|\tilde{\kappa}_{l}^{(E)}-\tilde{\kappa}\right\|^{2}}{2 \sigma_{n}^{2}}+\beta \sum_{j=1}^{J} \sum_{k, l} h_{n}\left((\mathcal{W} \tilde{\kappa})_{j, k, l}\right)$

where $\sigma_{n}$ the noise standard deviation in $\tilde{\kappa}_{l}^{(E)}, J$ the number of scales, $\beta$ is the regularization parameter and $\mathcal{W}$ is the Wavelet Transform operator. The $\beta$ parameter is calculated automatically under the constraint that the residual should have a standard deviation equal to the noise standard deviation. Full details of the minimization algorithm can be found in Starck et al. (2001), as well as the way to determine automatically the regularization parameter $\beta$.

\subsection{Related work}

\subsubsection{The generalized wavelet regularization}

Using a prior such that a pixel value is a function of its neighborhood (see Molina et al. 2001, for more details on the Markov Random Field model), the Bayesian solution consists in adding the following penalization on the solution:

$$
\begin{aligned}
C(\tilde{\kappa})= & \beta \sum_{x} \sum_{y}\left(\phi(\tilde{\kappa}(x, y)-\tilde{\kappa}(x, y+1))^{2}\right. \\
& \left.+\phi(\tilde{\kappa}(x, y)-\tilde{\kappa}(x+1, y))^{2}\right)^{\frac{1}{2}} .
\end{aligned}
$$

The function $\phi$, called potential function, is an edge preserving function. The term $\beta \sum_{x} \sum_{y} \phi(\|\nabla I\|(x, y))$ can also be interpreted as the Gibbs energy of a Markov Random Field. Generally, functions $\phi$ are chosen with a quadratic part which ensures a good smoothing of small gradients (Green 1990), and a linear behavior which cancels the penalization of large gradients (Bouman \& Sauer 1993):

1. $\lim _{t \rightarrow 0} \frac{\phi^{\prime}(t)}{2 t}=1$, smooth faint gradients;

2. $\lim _{t \rightarrow \infty} \frac{\phi^{\prime}(t)}{2 t}=0$, preserve strong gradients;

3. $\frac{\phi^{\prime}(t)}{2 t}$ is strictly decreasing.

Such functions are often called $L_{2}-L_{1}$ functions. Examples of $\phi$ functions:

1. $\phi_{\mathrm{q}}(x)=x^{2}$ : quadratic function;

2. $\phi_{\mathrm{TV}}(x)=|x|$ : Total Variation;

3. $\phi_{2}(x)=2 \sqrt{1+x^{2}}-2$ : Hyper-Surface (Charbonnier et al. 1997);

4. $\phi_{3}(x)=x^{2} /\left(1+x^{2}\right)($ Geman \& McClure 1985); 


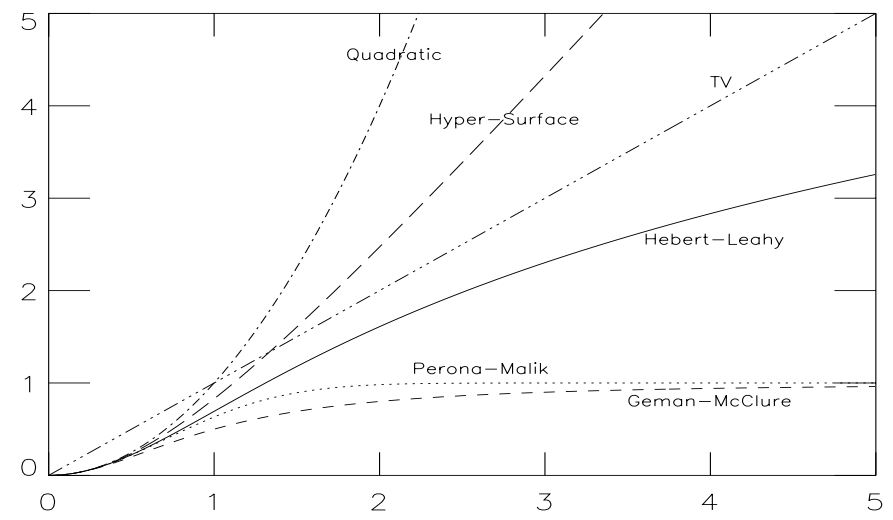

Fig. 4. Examples of potential function $\phi$.

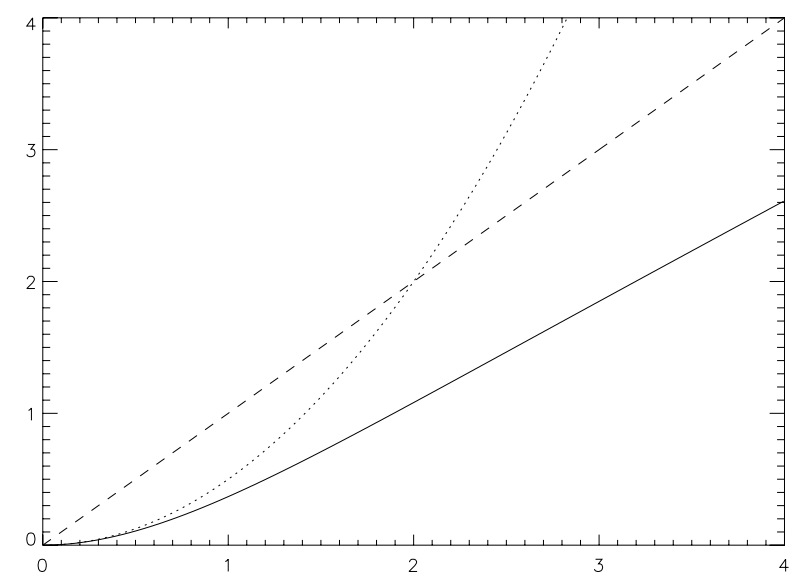

Fig. 5. Penalization functions: dashed, $l_{1}$ norm (i.e. $\phi(w)=|w|$ ); dotted $l_{2}$ norm $\phi(w)=\frac{w^{2}}{2}$ (i.e. ENERGY-MSE); continuous, multiscale entropy function (NOISE-MSE).

\section{5. $\phi_{4}(x)=1-e^{-x^{2}}($ Perona \& Malik 1990);}

6. $\phi_{5}(x)=\log \left(1+x^{2}\right)$ (Hebert \& Leahy 1989).

Figure 4 shows different $\phi$ functions.

It has been shown that this concept can be generalized in the wavelet domain, leading to a multiscale wavelet penalization term (Jalobeanu 2001):

$C_{w}(\tilde{\kappa})=\beta \sum_{j, k, l} \phi\left(\left\|(\mathcal{W} \tilde{\kappa})_{j, k, l}\right\|_{p}\right)$

When $\phi(x)=x$ and $p=1$, it corresponds to the $l_{1}$ norm of the wavelet coefficients. In this framework, the multiscale entropy deconvolution method is only one special case of the wavelet constraint deconvolution method.

Figure 5 shows the multiscale entropy penalization function. The dashed line corresponds to a $l_{1}$ penalization (i.e. $\phi(w)=|w|$ ), the dotted line to a $l_{2}$ penalization $\phi(w)=\frac{w^{2}}{2}$, and the continuous line to the multiscale entropy function. We can immediately see that the multiscale entropy function presents a quadratic behavior for small values, and is closer to the $l_{1}$ penalization function for large values. Penalization function with a $l_{2}-l_{1}$ behavior are known to be a good choice for image restoration.

\subsubsection{Multiscale MEM and ICF}

The multichannel ICF-MEM method (Weir 1991, 1992) consists in assuming that the visible-space image $O$ is formed by a weighted sum of the visible-space image channels $O_{j}$,
$O=\sum_{j=1}^{N_{\mathrm{c}}} p_{j} O_{j}$ where $N_{\mathrm{c}}$ is the number of channels and $O_{j}$ is the result of the convolution between a hidden image $h_{j}$ with a lowpass filter (ICF) $C_{j}$, called ICF (Intrinsic Correlation Function) (i.e. $O_{j}=C_{j} * h_{j}$ ). In practice, the ICF is a Gaussian. The MEM-ICF constraint is:

$C_{\mathrm{ICF}}=\sum_{j=1}^{N_{\mathrm{c}}}\left|h_{j}\right|-m_{j}-\left|h_{j}\right| \log \left(\frac{\left|h_{j}\right|}{m_{j}}\right)$.

In Maisinger et al. (2004), it was argued that the multiscale entropy is merely a special case of the intrinsic correlation function approach, where we replace the ICF kernel by a wavelet function. From the strict mathematical point of view, this is right, but this vision minimizes completely the improvement related to the wavelets. All the concepts of sparse representation (which is the key of the wavelet success in many applications), fast decomposition and reconstruction, zero mean coefficients (which allows us to get wavelet coefficients which are independent of the background and to derive a robust noise modeling) do not exist in the ICF-MEM approach. Furthermore, ICF-MEM approach requires to estimate accurately the background, which may be sometimes a very difficult task, and it has be shown (Bontekoe et al. 1994) that the solution depends strongly on this estimation. On the contrary, Multiscale MEM needs only an estimation of the noise standard deviation, which is easy to determine.

For all these reasons, we prefer to keep our vision of the multiscale entropy method as a specific case of the generalized wavelet regularization techniques rather than as an extension of the ICF approach.

\section{Results}

\subsection{Comparison of methods}

We have used a simulated data set obtained using a standard $\Lambda$-CDM cosmological model. A part of the $\kappa$ mass map and the shear maps is shown in Fig. 1. The field size is $2 \times 2$ square degrees, sampled with $1024 \times 1024$ pixels.

Noisy shear maps, corresponding to both spatial (i.e. $n_{\mathrm{g}}=$ 100 gals amin $^{-2}$ ) and ground-based observations (i.e. $n_{\mathrm{g}}=$ 20 gals amin ${ }^{-2}$ ), are created using Eq. (7). Then we have reconstructed the two noisy mass maps from Eq. (9) and applied the following methods:

1. Gaussian filtering with a standard deviation equal to $\sigma_{\mathrm{G}}=$ 1 amin.

2. Gaussian filtering with a standard deviation equal to $\sigma_{\mathrm{G}}=$ 2.5 amin.

3. Wiener filtering.

4. Maximum Entropy Method (MEM) using the LensEnt2 package. As this code has not been designed for manipulating large images, we had to restrict the restoration by this method to a field size of $0.5 \times 0.5$ square degree, sampled with $256 \times 256$ pixels. Since the LensEnt 2 maps are positivity constrained, as recommended by the author of the LensEnt 2 package, we have recovered a physical mass by transforming the outputs such that the minimum convergence in the central quarter of the reconstruction is zero. To optimize the ICF, we have maximized the Bayesian evidence value as a function of ICF width, and found that maximum evidence is around 210 arcsec for ground observations and around 180 arcsec for space observations.

5. Multiscale Entropy method. 
Table 1. Standard deviation of the reconstruction error with five different methods.

\begin{tabular}{c|c|c}
\hline \hline Method & Error $\left(n_{\mathrm{g}}=20 \mathrm{gal} / \mathrm{amin}^{2}\right)$ & Error $\left(n_{\mathrm{g}}=100 \mathrm{gal} / \mathrm{amin}^{2}\right)$ \\
\hline Gaussian Filtering $\left(\sigma_{\mathrm{G}}=1 \mathrm{amin}\right)$ & 1.108 & 0.775 \\
Gaussian Filtering $\left(\sigma_{\mathrm{G}}=2.5 \mathrm{amin}\right)$ & 0.9138 & 0.868 \\
Wiener Filtering & 0.888 & 0.770 \\
MEM-LensEnt2 & 1.091 & 0.821 \\
Multiscale Entropy Filtering & 0.888 & 0.746 \\
\hline
\end{tabular}
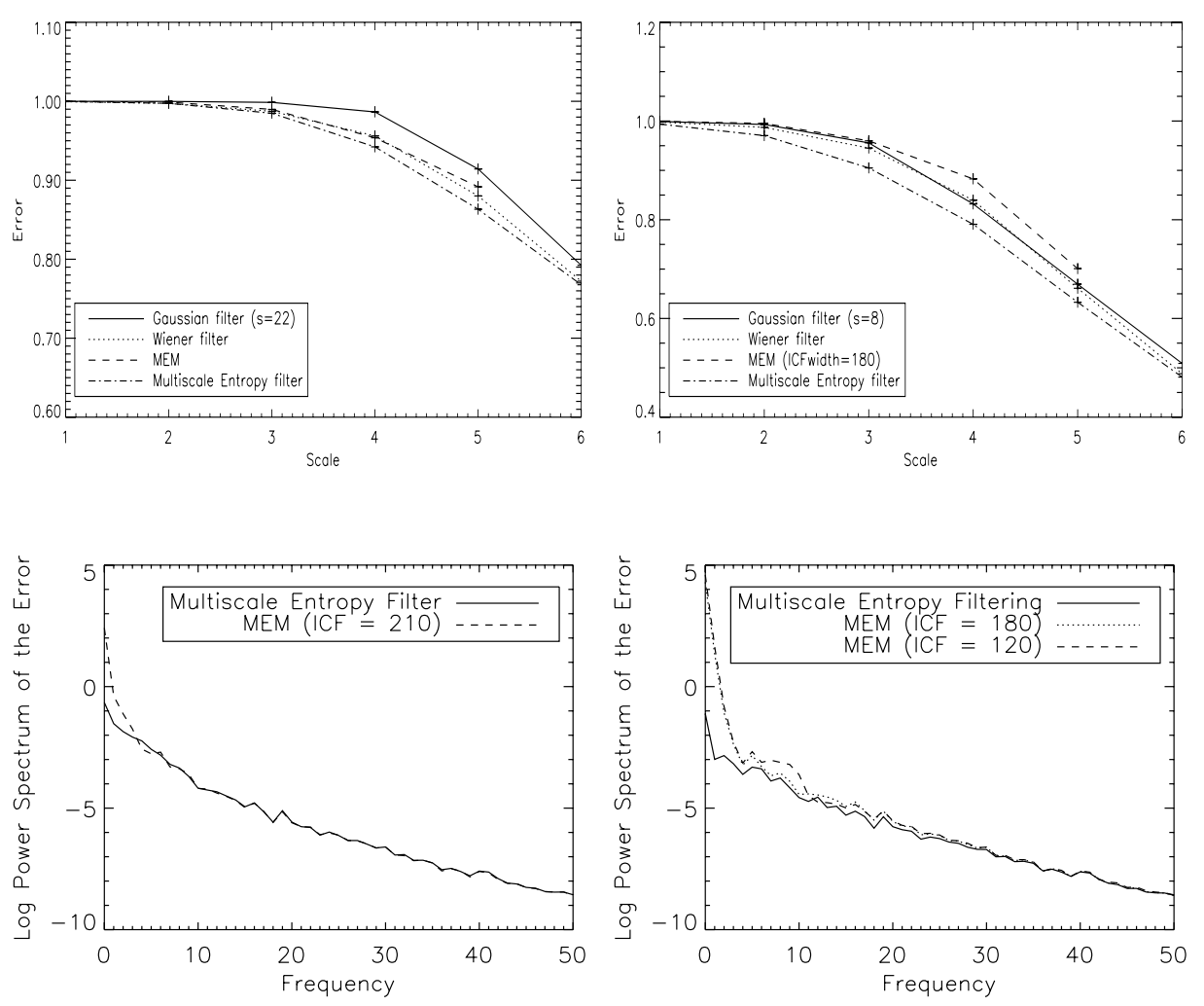

Fig. 6. Standard deviation versus scale for the ground-based simulation (left) and the spacebased simulation (right).

Fig. 7. Log Power Spectrum of the Error by Multiscale Entropy Filter and MEM for the ground-based simulation (left) and the spacebased simulation (right).
The evaluation is done by i) visual inspection of the images, ii) calculating the standard deviation between the original $\kappa$ mass map and the reconstructed map (i.e. $\left.E=\frac{\operatorname{STD}(\kappa-\widetilde{\kappa})}{\operatorname{STD}(\kappa)}\right)$, iii) calculating the standard deviation for each of their wavelet scales (i.e. $\left.E_{j}=\frac{\operatorname{STD}\left((\mathcal{W} \kappa)_{j}-(\mathcal{W} \tilde{\kappa})_{j}\right)}{\operatorname{STD}\left((\mathcal{W} \kappa)_{j}\right)}\right)$ and iv) calculation the power spectrum of the error $E$ (for MEM and multiscale entropy methods).

The values $\sigma_{\mathrm{G}}=1$ amin and $\sigma_{\mathrm{G}}=2.5$ amin have been chosen to optimize the Gaussian filtering for $n_{\mathrm{g}}=100 \mathrm{gals} \mathrm{arcmin}^{-2}$ and $n_{\mathrm{g}}=20$ gals $\operatorname{arcmin}^{-2}$, respectively. Table 1 gives the standard deviation of the error for the four reconstructed mass maps. It shows that i) the Wiener filtering is better than the Gaussian filtering and the MEM-LensEnt2 method and ii) the Multiscale Entropy outperforms the three other methods.

Figure 6 shows the error versus the scale (each wavelet scale) for both simulations using the Gaussian filtering (continuous line), the Wiener filtering (dotted line), the MEM-LensEnt2 filtering (dashed line) and the Multiscale Entropy filtering (dotteddashed line). The wavelet scales 1 to 6 correspond to scales of $0.12,0.23,0.47,0.94,1.87,3.75$ amin respectively. We can see that the Multiscale Entropy method produces better results for all scales.

Figure 7 shows the log power spectrum of the error. It is very consistent with the previous one. Indeed, the MEM error becomes very important toward the smallest frequencies (largest wavelet scales). The same experiment has been done with a smallest ICF (ICF $=120$ for the spatial simulation), but the result is worse, which is not surprising since the ICF value was chosen to get the best results.

Figure 8 shows from top to bottom the reconstructed maps for the Gaussian, the Wiener and Multiscale Entropy filtering. Figure 8 left corresponds to ground-based observations (i.e. $n_{\mathrm{g}}=$ 20) and Fig. 8 right corresponds to spatial observations (i.e. $n_{\mathrm{g}}=$ 100).

Figure 9 shows the denoising results on a portion of the previous image. Figure 9 shows the original noise free simulated image of the $0.5 \times 0.5$ square degrees field (upper left), the Multiscale Entropy Filtering for the spatial simulated observations $\left(n_{\mathrm{G}}=100\right)$ (upper right), the MEM-LensEnt2 restoration for the ground based observations (bottom left) and the spatial observations (bottom right).

The computation time for the $1024 \times 1024$ pixels map is $4 \mathrm{~min}$ for the Multiscale Entropy method, $26 \mathrm{~s}$ for the Wiener filtering and $4 \mathrm{~s}$ for the Gaussian smoothing. The computation time for the $256 \times 256$ pixels map is around 60 min (it depends on the convergence of the result) using the MEM-LensEnt2 package. 

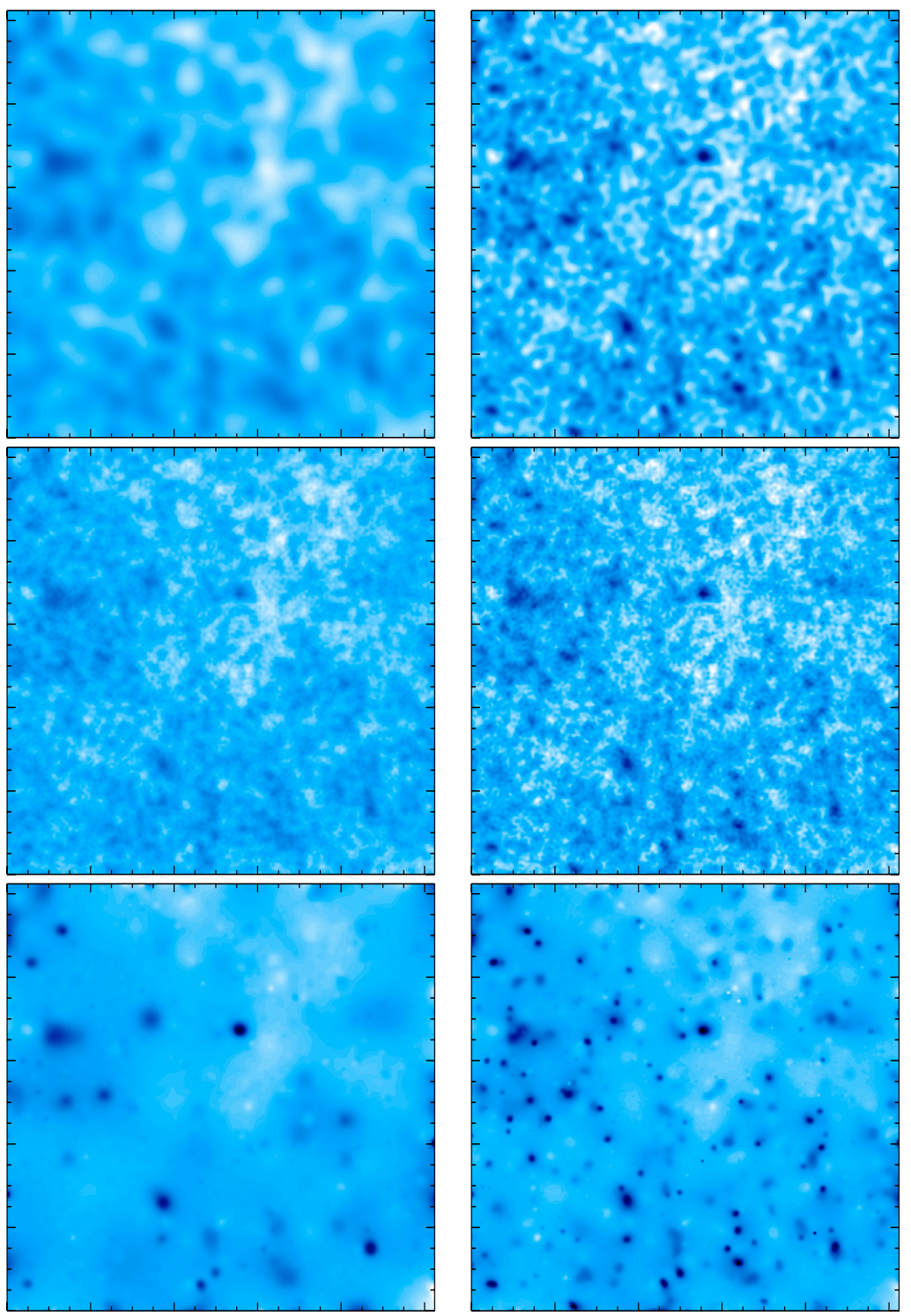

Fig. 8. Restoration of the $2 \times 2$ square degrees ground-based observation (left) and spatial observation (right). From top to bottom, Gaussian filtering, Wiener filtering and Multiscale Entropy filtering.

\subsection{Robustness to missing data}

During the observations, various problem can cause a loss of data in the image. For example, it can be due to a defect of the camera CCD, generating a dark line or a dark row in the image, or to the presence of a bright star in the field of view which forces us to remove part of the image. In order to study this problem, we mask two rectangular areas, setting all pixel values to 0 , in the shear maps $\gamma_{1}$ and $\gamma_{2}$. By inverse filtering, we have derived the noisy mass map $\kappa_{l}$ in which we can also visualize the lack of data (Fig. 10 upper left). Then we have applied the three methods, Gaussian filtering, Wiener filtering and Multiscale Entropy, to the noisy mass map and the results can be seen respectively in Fig. 10 upper right, Fig. 10 bottom left and bottom right. We can see that all three methods are robust to the missing data. Note however that, for the Wiener filtering, we have assumed perfect knowledge of the power spectrum of $\kappa$, while, in practice, its estimation is made more complicated by the complex field geometry.

Figure 11 shows the error versus the scale for both simulations using the Gaussian filtering (continuous line), the Wiener filtering (dotted line) and Multiscale Entropy. We can see that the Multiscale Entropy still produces better results at all scales. Bayesian methods such MEM could also take into account properly missing data, however not in a straightforward way as when using wavelets.

\subsection{Cluster detection}

Another important aspect of the weak shear mass reconstruction is the possibility to detect clusters and to build a catalog. Here, using the FDR in the wavelet space, we detect as significant a set of wavelet coefficients. We built an isophote map, where each isophote level corresponds to the detection level in a given scale. This isophote is overplotted on the true mass map, which allows us to visually check the false detections and the missed detections. A cluster surrounded by two isophotes means that it has been detected at two scales. Figure 12 left shows the isophote map when we use the regular $k \sigma$ thresholding and Fig. 12 left right shows the isophote map when we use the FDR method. We see that the FDR is more sensitive than the $k \sigma$ method for the 

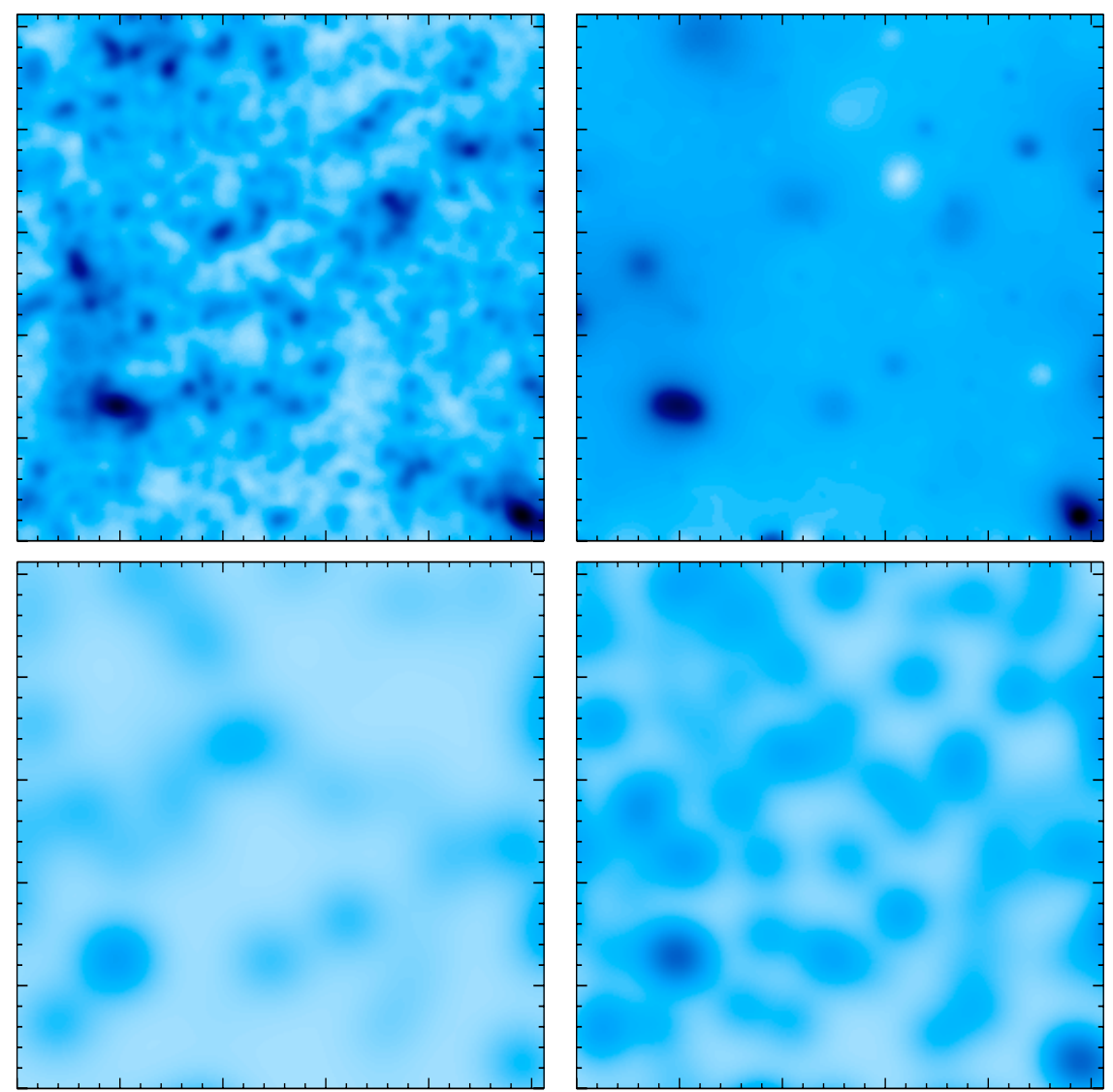

Fig. 9. In a region of $0.5 \times 0.5$ square degrees, a sixteenth of the original field: upper left, simulated mass map, upper right, Multiscale entropy filtering for $n_{\mathrm{g}}=100 \mathrm{gal} / \mathrm{arcmin}^{2}$. Bottom left, MEM filtering for $n_{\mathrm{g}}=20 \mathrm{gals} / \mathrm{amin}^{-2}$ (ICF width $=$ 210) and bottom right for $n_{\mathrm{g}}=100 \mathrm{gals} / \mathrm{amin}^{-2}$ $(\mathrm{ICF}$ width $=180)$.
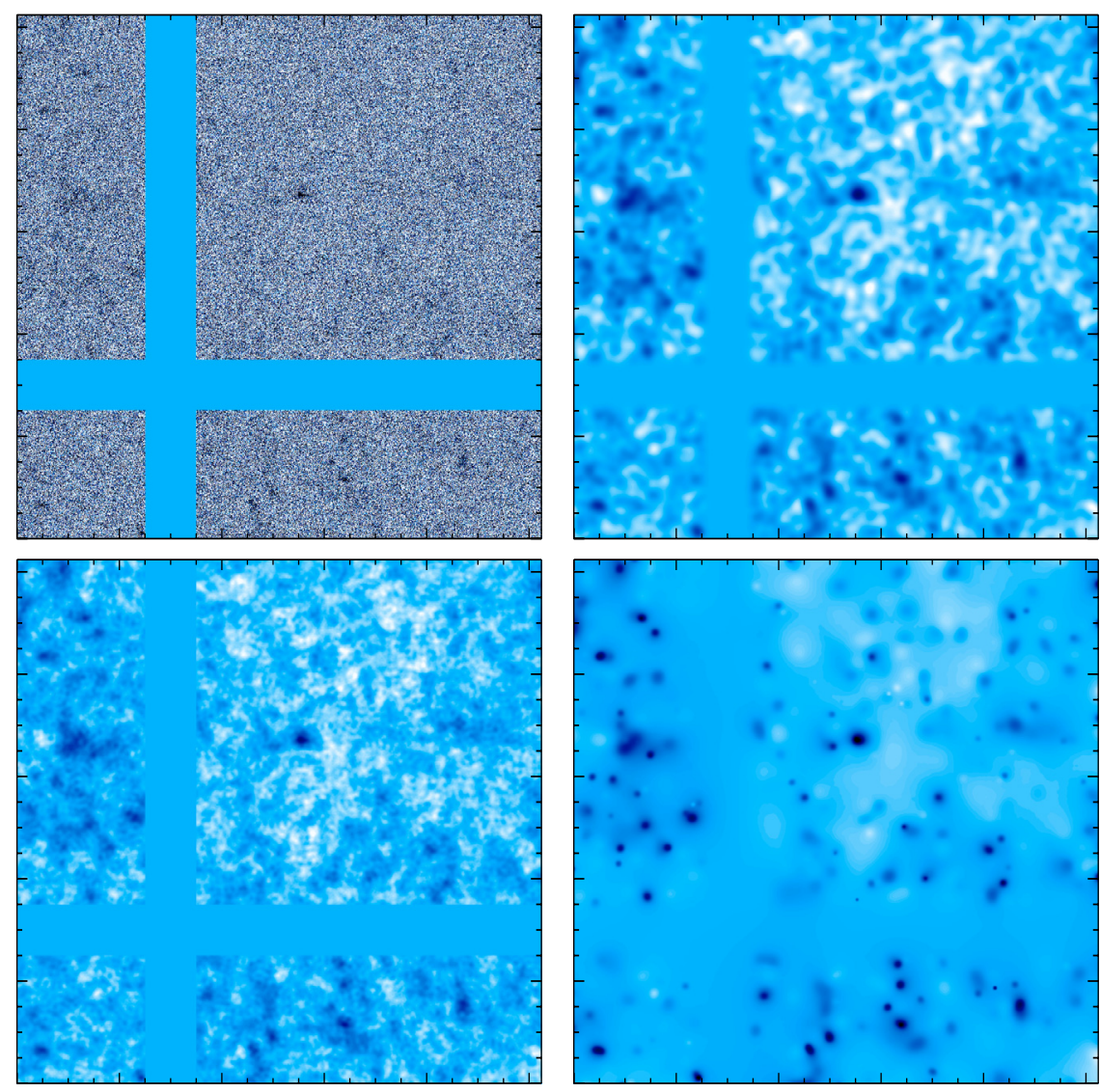

Fig. 10. Upper left, noisy shear map $\left(n_{\mathrm{g}}=\right.$ $\left.100 \mathrm{gal} / \mathrm{arcmin}^{2}\right)$. Upper right, Gaussian filtering. Bottom left, Wiener filtering, and bottom right, Multiscale Entropy filtering. 

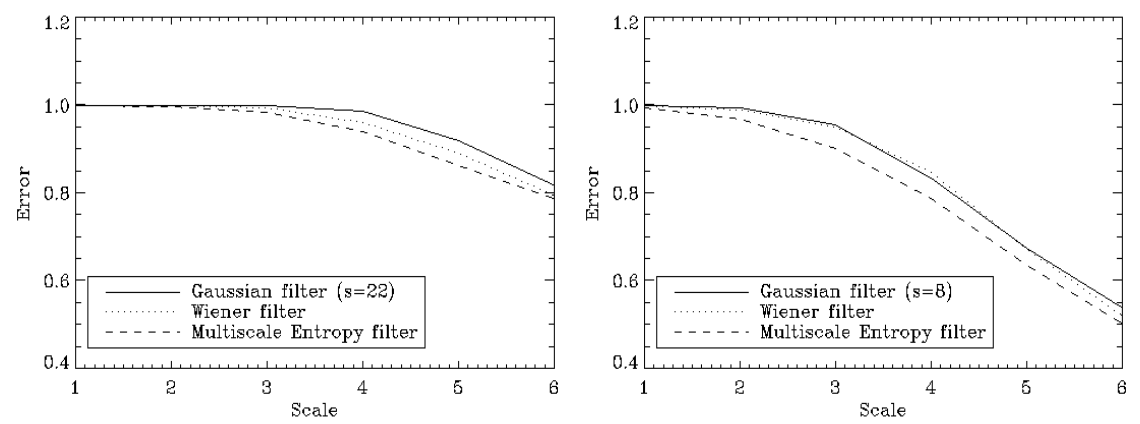

Fig. 11. Standard deviation versus scale for the ground-based simulation (left) and the spatial simulation (right) with missing data.
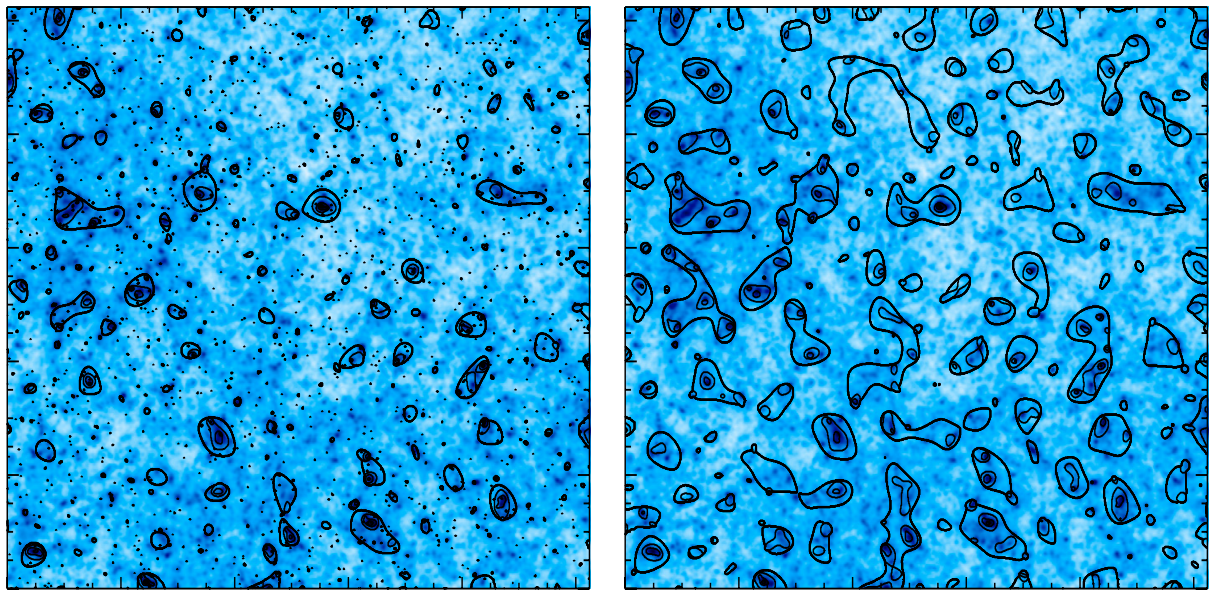

Fig. 12. Isophotes of the detected wavelet coefficients for the space-based simulation overplotted on the original mass map: left, using the $k \sigma$ standard approach (with $k=3,4,5$ ) and right using the FDR method.
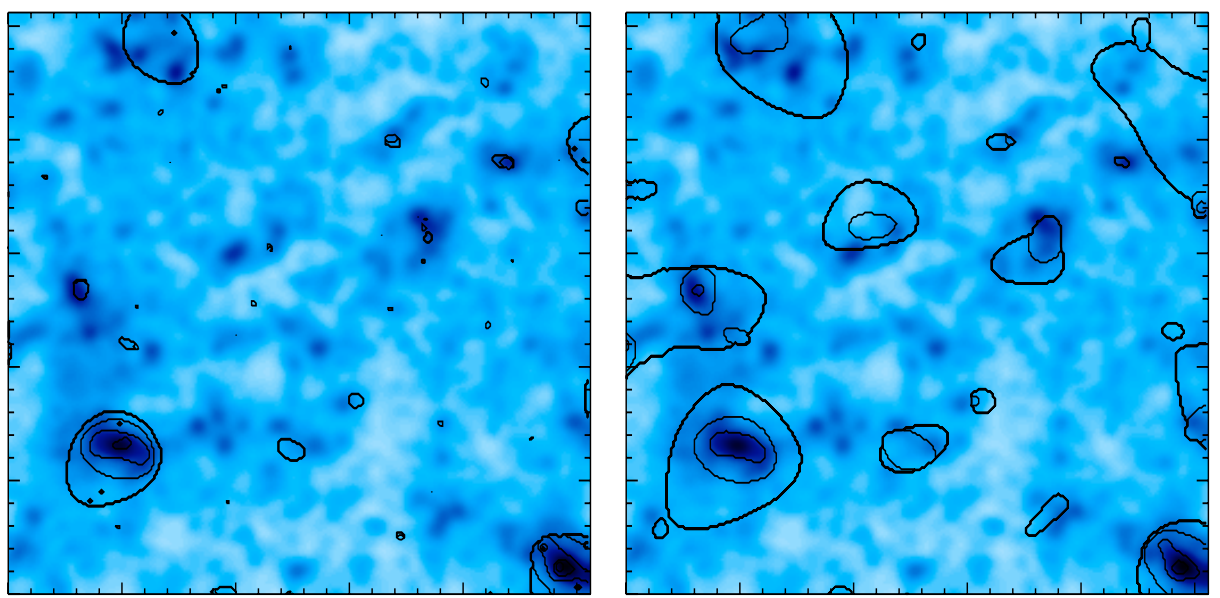

Fig. 13. Zoom of the previous maps.

detection, without being contaminated by a large number of false detections. Figure 13 shows a zoom of these two maps. Figure 14 shows a comparison between the Gaussian filtering, the Wiener filtering and FDR-Wavelet method for the detection of clusters. In the Gaussian and Wiener maps, the isophotes corresponds to a $k \sigma$ detection level where $k=3,4,5$. It shows clearly how the FDR-Wavelet method outperforms the other methods.

\subsection{E/B Decomposition}

As explained in Sect. 2.3, a simple diagnostic test for a wide range of systematic effects is to search for the presence of
B-mode in the lensing maps. In order to test it, we have simulated mass maps with a B-mode.

Figure 15 left shows a simulated mass map with a lensing E-mode signal (left) and an arbitrary B-mode signal (left). As usual, we have added a realistic space-based Gaussian noise to the shear of this simulation. Figure 16 shows the noisy mass map resulting. Using the Multiscale Entropy filtering, we have then reconstructed the two components of the mass map (see Sect. 2.3): E-mode in Fig. 17 left and B-mode in Fig. 17 right. We see clearly that the wavelet separation of the E and B modes is very good. Indeed, the two main features in the B-mode have well been recovered, without interfering with the reconstruction of the E-mode. 

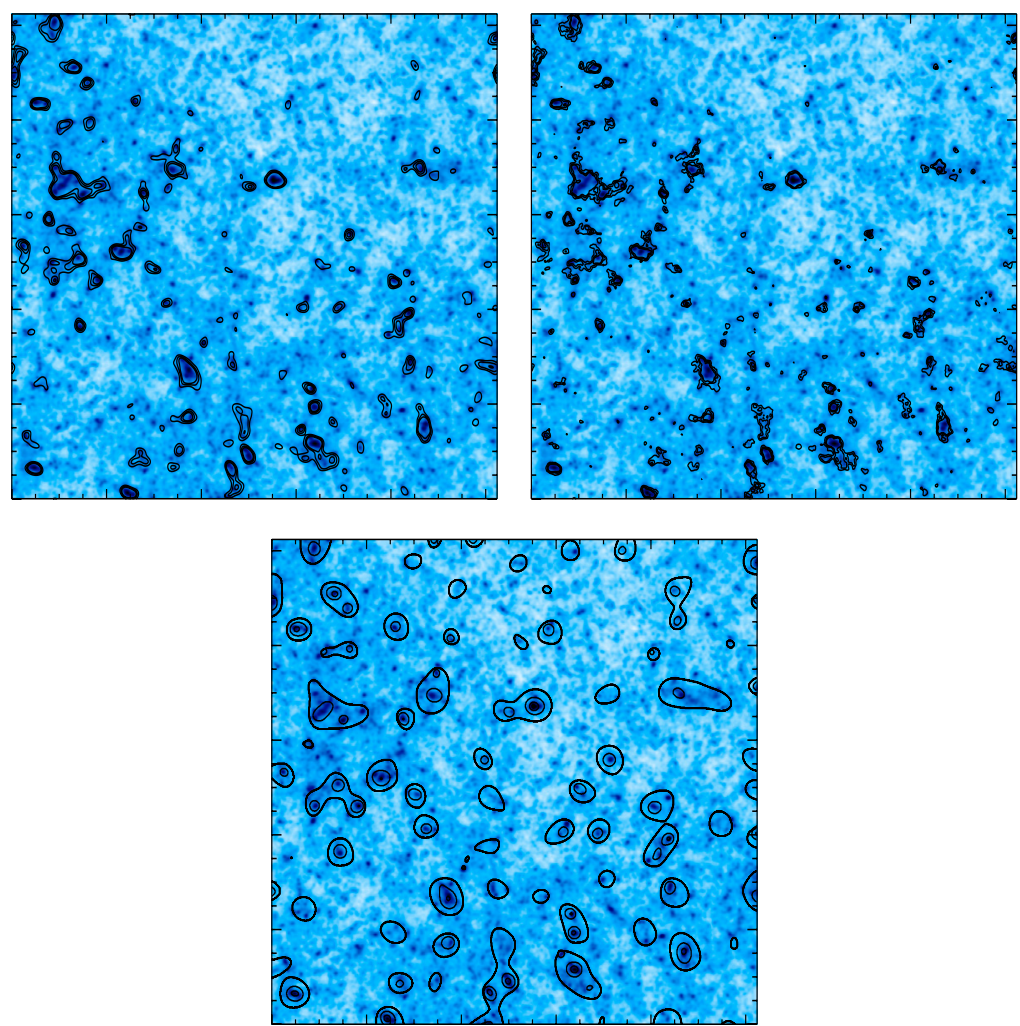

Fig. 14. The isophotes represent the detected clusters using the Gaussian filtering (upper left), the Wiener filtering (upper right) and the wavelet-FDR method.
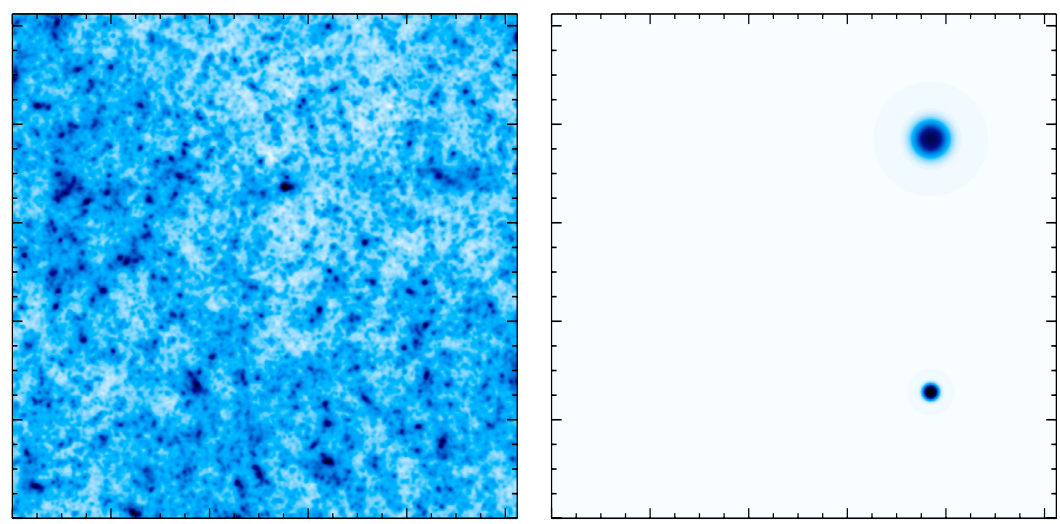

Fig. 15. left mass map (E-mode), right mass map (B-mode).

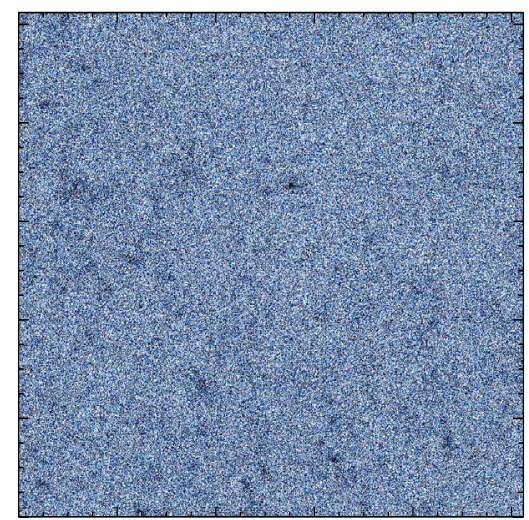

\section{Conclusion}

We have presented in this paper a new way to reconstruct weak lensing mass maps. We have modified the Multiscale Entropy method in order to take into account the FDR. We have shown that this new method outperforms several standard techniques currently used for the weak shear mass reconstruction. The visual aspect as well as objective criteria, such the rms of the error or the rms per scale of the error, clearly show the advantages of the proposed approach. Experiments have demonstrated that it is also robust to missing data. We have also shown that a $\mathrm{E} / \mathrm{B}$ mode separation can also be performed using this method, thus providing a useful test for the spatial distribution of residual systematics. Our method allows us also to build a catalog of clusters and the use of FDR leads to a clear improvement in sensitivity, compared to what has been done previously with wavelets.

Fig. 16. Noisy simulated mass map. 

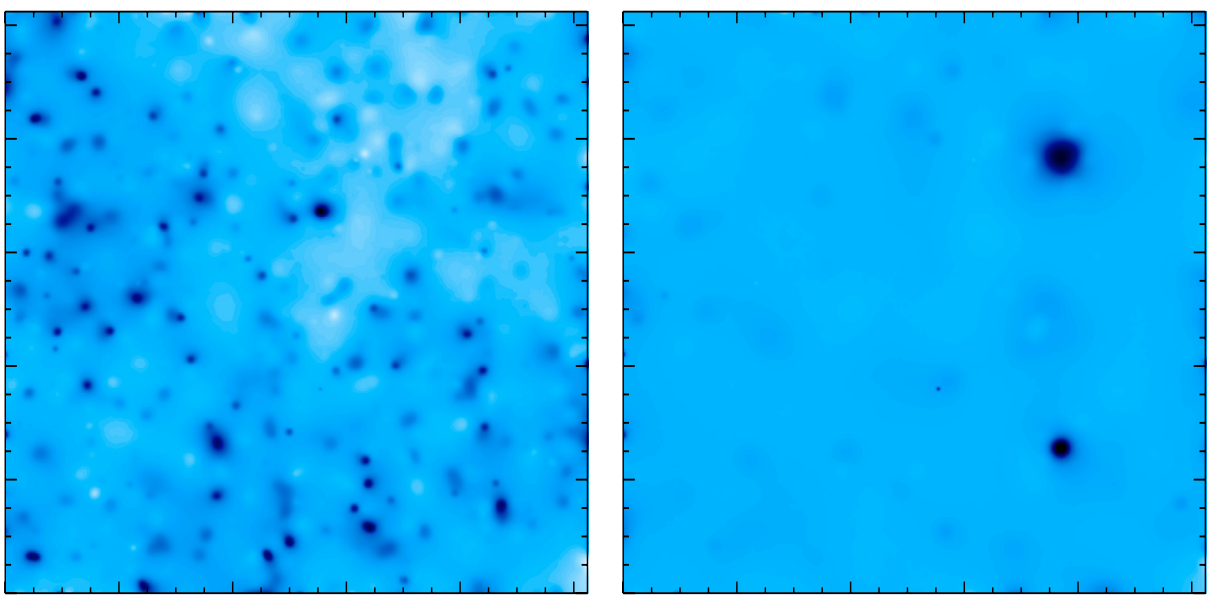

Fig. 17. left filtered noisy mass map (E-mode), right filtered noisy mass map (B-mode).

\section{Software}

The software related to this paper, MR/Lens, and its full documentation are available from the following web page: http://jstarck. free. fr/mrlens.hmtl

Acknowledgements. We wish to thank Savita Mathur for her initial work on the subject, Richard Massey and Bedros Afeyan for useful discussions. We would like to particularly acknowledge Phil Marshall for his help relative to the use of the LensEnt2 package.

\section{References}

Bartelmann, M. 1995, A\&A, 303, 643

Bartelmann, M., \& Schneider, P. 1999, ArXiv Astrophysics e-prints

Bartelmann, M., Narayan, R., Seitz, S., \& Schneider, P. 1996, ApJ, 464, L115

Benjamini, Y., \& Hochberg, Y. 1995, J. R. Stat. Soc. B, 57, 289

Bontekoe, T., Koper, E., \& Kester, D. 1994, A\&A, 284, 1037

Bouman, C. A., \& Sauer, K. 1993, IEEE Trans. Image Proc., 2, 296

Bridle, S. L., Hobson, M. P., Lasenby, A. N., \& Saunders, R. 1998, MNRAS, 299, 895

Charbonnier, P., Blanc-Feraud, L., Aubert, G., \& Barlaud, M. 1997, IEEE Trans. Image Proc., 6, 298

Donoho, D. L., \& Elad, M. 2003, Proc. Nat. Aca. Sci., 100, 2197

Geman, S., \& McClure, D. 1985, in Proc. Statist. Comput. Sect., ed. A. S. Assoc., Washington DC

Genovese, C. R., Noll, N. C., \& Eddy, W. F. 1997, Magn. Res. Med., 38, 497

Green, P. J. 1990, IEEE Trans. Medical Imaging, 9, 84

Gull, S., \& Skilling, J. 1991, MEMSYS5 Quantified Maximum Entropy User's Manual, Royston, England
Hebert, T., \& Leahy, R. 1989, IEEE Trans. Medical Imaging, 8, 194 Hopkins, A. M., Miller, C. J., Connolly, A. J., et al. 2002, AJ, 123, 1086 Jalobeanu, A. 2001, Ph.D. Thesis, Université de Nice Sophia Antipolis Kaiser, N. 1995, ApJ, 439, L1

Kaiser, N., \& Squires, G. 1993, ApJ, 404, 441

Maisinger, K., Hobson, M. P., \& Lasenby, A. N. 2004, MNRAS, 347, 339

Marshall, P. J., Hobson, M. P., Gull, S. F., \& Bridle, S. L. 2002, MNRAS, 335, 1037

Mellier, Y. 1999, ARA\&A, 37, 127

Mellier, Y. 2002, Space Sci. Rev., 100, 73

Miller, C. J., Genovese, C., Nichol, R. C., et al. 2001, AJ, 122, 3492

Molina, R., Núñez, J., Cortijo, F., \& Mateos, J. 2001, IEEE Signal Processing Magazine, 18, 11

Murtagh, F., Starck, J.-L., \& Bijaoui, A. 1995, A\&AS, 112, 179

Narayan, R. \& Nityananda, R. 1986, ARA\&A, 24, 127

Pantin, E., \& Starck, J.-L. 1996, A\&AS, 315, 575

Perona, P., \& Malik, J. 1990, IEEE Trans. Pattern Analysis and Machine Intelligence, 12, 629

Refregier, A. 2003, ARA\&A, 41,645

Schneider, P., \& Seitz, C. 1995, A\&A, 294, 411

Seitz, S., Schneider, P., \& Bartelmann, M. 1998, A\&A, 337, 325

Squires, G., Kaiser, N. 1996, ApJ, 473, 65

Starck, J.-L., \& Murtagh, F. 2002, Astronomical Image and Data Analysis (Springer-Verlag)

Starck, J.-L., Murtagh, F., \& Bijaoui, A. 1998, Image Processing and Data Analysis: The Multiscale Approach (Cambridge University Press)

Starck, J.-L., Murtagh, F., Querre, P., \& Bonnarel, F. 2001, A\&A, 368, 730 Starck, J.-L., Pantin, E., \& Murtagh, F. 2002, Publ. Astron. Soc. Pacific, 114, 1051

Vale, C., \& White, M. 2003, ApJ, 592, 699

Van Waerbeke, L., Mellier, Y., Radovich, M., et al. 2001, A\&A, 374, 757

Weir, N. 1991, in 3rd ESO/ST-ECF Data Analysis Workshop

Weir, N. 1992, in Astronomical Data Analysis Software and System 1, ed. D. Worral, C. Biemesderfer, \& J. Barnes, 186 (ASP) 